\title{
Reply to a Letter to the Editor
}

\author{
Motonobu Kimizuka $^{1} \cdot$ Michiaki Yamakage $^{1}$
}

Received: 24 February 2021 / Accepted: 4 March 2021 / Published online: 17 March 2021

(c) Japanese Society of Anesthesiologists 2021

To the Editor:

Thank you for your interest in our research and for your valuable comments.

Regarding the first comment, our objective was to investigate which type of anesthetic is appropriate as general anesthesia or sedative after C-section from the perspective of preventing atonic hemorrhage and to determine the mechanism. We believe that this is an extremely important research topic also from the perspective of clinical anesthesia. Moreover, because the activity of uterine smooth muscle, similar to that of other airway or vascular smooth muscles, is affected by factors such as autonomic nerves and humoral factors, it had always been an issue to determine what is important to monitor from the perspective of "atonic hemorrhage" by conducting in vivo studies. As you have indicated, the method to measure the contraction capacity of uterine smooth muscle in vivo has yet to be established; the only report from another study is a method using a three-dimensional pressure-velocity (slope) topogram [1]. As pointed out, future validation is needed to determine whether this method is the most appropriate approach, accurately reflecting the uterine muscle contraction in vivo. In this sense, we believe that our approach with a balloon, albeit self-made, is the first study that directly reflects the contraction pressure within the uterus.

Regarding the next comment, many studies of smooth muscle contraction induce depolarization with a high concentration of $\mathrm{KCl}$ and measure contraction with non-receptor mediated, simple $\mathrm{Ca}^{2}$ influx alone. However, during actual C-section, oxytocin or prostaglandin-induced uterine contraction occurs, leading to a question of whether it is appropriate to use $\mathrm{KCl}$-induced

This reply refers to the comment available online at https://doi. org/10.1007/s00540-021-02919-7.

Michiaki Yamakage

yamakage@sapmed.ac.jp

1 Department of Anesthesiology, Sapporo Medical University School of Medicine, South-1, West-16, Chuo-ku, Sapporo, Hokkaido 060-8543, Japan contraction as a reference. This is because, as you may know, the uterine smooth muscle contraction via receptor stimulation or automaticity, unlike vascular smooth muscle or airway smooth muscle, is periodic, and the contraction capacity often depends not only on the amplitude and frequency, but also on the AUC. Thus, when contraction with $\mathrm{KCl}$ is used, it may also be difficult to determine what to use as a reference.

Regarding the comment on the discussion of the difference between in vivo and in vitro studies, we believe this is described in detail in the manuscript. This difference is indeed the foundation and purpose of this study, and is the reason why the arachidonic acid cascade mechanism could not be found with in vitro studies of dexmedetomidine alone.

In the final comment regarding the use of a relatively higher glucose concentration compared to other smooth muscle studies, unlike other smooth muscles, uterine smooth muscle that has automaticity requires a greater amount of glucose to maintain stable contraction strength under the same environment as body temperature and in the Krebs condition. We believe this is similar to when attempting to stably attain electrical activity using sliced specimens of the hippocampus and brain stem, which requires finding the optimal conditions by changing temperature, animal age, $\mathrm{KCl}$ concentration, and glucose concentration [2].

Thank you again for your interest in our study and for your positive and insightful comments. We plan to incorporate these in the further development of our studies.

\section{References}

1. Smith RP. Pressure-velocity analysis of uterine muscle during spontaneous dysmenorrheic contractions in vivo. Am J Obstet Gynecol. 1989;160:1400-3.

2. Nomura K, Narimatsu E, Inoue H, Kyan R, Sawamoto K, Uemura S, Kakizaki R, Harada K. Mechanism of central hypopnea induced by organic phosphorus poisoning. Sci Rep. 2021. https://doi. org/10.1038/s41598-020-73003-5.

Publisher's Note Springer Nature remains neutral with regard to jurisdictional claims in published maps and institutional affiliations. 